Supporting Information

Ascospiroketals A and B, unprecedented cycloethers from the marine-derived fungus Ascochyta salicorniae

Simon F. Seibert, Anja Krick, Ekaterina Eguereva, Stefan Kehraus, and Gabriele M. König*

Institute for Pharmaceutical Biology, University of Bonn, Nussallee 6, D-53115 Bonn, Germany

General Experimental Procedures

Isolation and Taxonomy of the Fungus

\title{
Cultivation
}

\section{Extraction and Isolation}

\section{Spectral Data for Ascospiroketal A (1)}

Table S1 $\quad{ }^{1} \mathrm{H}$ and ${ }^{13} \mathrm{C}$ NMR spectral data for Ascospiroketal A (1) in $\left(\mathrm{CD}_{3}\right)_{2} \mathrm{CO}$

Figure $\mathrm{S} 1 \quad 500 \mathrm{MHz}{ }^{1} \mathrm{H}$ NMR spectrum of Ascospiroketal A (1) in $\left(\mathrm{CD}_{3}\right)_{2} \mathrm{CO}$

Figure S2 $\quad 75 \mathrm{MHz}{ }^{13} \mathrm{C}$ NMR and $75 \mathrm{MHz}$ DEPT 135 spectra of Ascospiroketal A (1) in $\left(\mathrm{CD}_{3}\right)_{2} \mathrm{CO}$

Figure $\mathrm{S} 3 \quad 500 \mathrm{MHz}{ }^{1} \mathrm{H}-{ }^{1} \mathrm{H}-\mathrm{COSY}$ spectrum of Ascospiroketal A (1) in $\left(\mathrm{CD}_{3}\right)_{2} \mathrm{CO}$

Figure $\mathrm{S} 4 \quad 500 \mathrm{MHz}$ HSQC spectrum of Ascospiroketal A (1) in $\left(\mathrm{CD}_{3}\right)_{2} \mathrm{CO}$

Figure S5 $500 \mathrm{MHz}$ HMBC spectrum of Ascospiroketal A (1) in $\left(\mathrm{CD}_{3}\right)_{2} \mathrm{CO}$

Figure S6 $500 \mathrm{MHz}$ NOESY spectrum of Ascospiroketal A (1) in $\left(\mathrm{CD}_{3}\right)_{2} \mathrm{CO}$

Figure S7 3D drawing of Ascospiroketal A (1) with selected NOESY correlations

\section{Spectral Data for Ascospiroketal B (2)}

Table S2 $\quad{ }^{1} \mathrm{H}$ and ${ }^{13} \mathrm{C}$ NMR spectral data for Ascospiroketal B (2) in $\left(\mathrm{CD}_{3}\right)_{2} \mathrm{CO}$

Figure S8 $\quad 500 \mathrm{MHz}{ }^{1} \mathrm{H}$ NMR spectrum of Ascospiroketal B (2) in $\left(\mathrm{CD}_{3}\right)_{2} \mathrm{CO}$

Figure S9 $\quad 75 \mathrm{MHz}{ }^{13} \mathrm{C}$ NMR and $75 \mathrm{MHz}$ DEPT 135 spectra of Ascospiroketal B (2) in $\left(\mathrm{CD}_{3}\right)_{2} \mathrm{CO}$

Figure S10 $500 \mathrm{MHz}{ }^{1} \mathrm{H}-{ }^{1} \mathrm{H}-\mathrm{COSY}$ spectrum of Ascospiroketal B (2) in $\left(\mathrm{CD}_{3}\right)_{2} \mathrm{CO}$

Figure S11 $500 \mathrm{MHz}$ HSQC spectrum of Ascospiroketal B (2) in $\left(\mathrm{CD}_{3}\right)_{2} \mathrm{CO}$

Figure S12 $500 \mathrm{MHz}$ HMBC spectrum of Ascospiroketal B (2) in $\left(\mathrm{CD}_{3}\right)_{2} \mathrm{CO}$

Figure S13 $500 \mathrm{MHz}$ NOESY spectrum of Ascospiroketal B (2) in $\left(\mathrm{CD}_{3}\right)_{2} \mathrm{CO}$

Figure S14 3D drawing of Ascospiroketal B (2) with selected NOESY correlations

General Experimental Procedures. Optical rotations were measured with a Jasco DIP 140 polarimeter. UV and IR spectra were obtained employing Perkin-Elmer Lambda 40 and Perkin-Elmer Spectrum BX instruments, respectively. All NMR spectra were recorded on Bruker Avance 300 DPX and Bruker Avance 500 DRX spectrometers in $\left(\mathrm{CD}_{3}\right)_{2} \mathrm{CO}$. Spectra were referenced to residual solvent signals with resonances at $\delta_{\mathrm{H} / \mathrm{C}} 2.04 / 29.8 \mathrm{ppm}$. EI-MS were recorded using a Finnigan MAT 95 spectrometer. HPLC was carried out using a MerckHitachi system consisting of an L-6200 A pump, an L-4500 A photodiode array detector and a D-6000 A interface, together with a Knauer K-2300 differential refractometer as detector. HPLC columns were from Phenomenex [column (1): Luna 100-5, C-18, 250 x 10 mm] and 
from Macherey-Nagel [column (2): Nucleodur 100-5, C-18 EC, 250 x 4.6 mm]. Polygoprep 60-50 $\mathrm{C}_{18}$ from Macherey-Nagel has been used for vacuum liquid chromatography (VLC). Sephadex LH-20 material for column chromatography was obtained from Amersham Pharmacia Biotech AB.

Isolation and Taxonomy of the Fungus. Isolation and taxonomy of the fungus have been previously described by Osterhage et al. 2000.

Cultivation. The fungus was cultivated in Fernbach flasks on a solid medium containing 20 $\mathrm{g} / \mathrm{L}$ biomalt ${ }^{\circledR}$ extract (Villa Natura, Kirn, Germany), $15 \mathrm{~g} / \mathrm{L}$ agar and $80 \%$ artificial seawater at room temperature for 53 and 68 days. The artificial seawater contained $(\mathrm{g} / \mathrm{L}): \mathrm{KBr}(0.1)$, $\mathrm{NaCl}$ (23.48), $\mathrm{MgCl}_{2} * 6 \mathrm{H}_{2} \mathrm{O}$ (10.61), $\mathrm{CaCl}_{2} * 2 \mathrm{H}_{2} \mathrm{O}$ (1.47), $\mathrm{KCl}(0.66), \mathrm{SrCl}_{2} * 6 \mathrm{H}_{2} \mathrm{O}$ (0.04), $\mathrm{Na}_{2} \mathrm{SO}_{4}$ (3.92), $\mathrm{NaHCO}_{3}(0.19)$ and $\mathrm{H}_{3} \mathrm{BO}_{3}(0.03)$.

Extraction and Isolation. Cultivation medium $(25 \mathrm{~L})$ and mycelia were extracted with ethyl acetate $(6 \times 25 \mathrm{~L})$ after being homogenized using an Ultra Turrax T45. The ethyl acetate extract (10.7 g, brown oil) was fractionated by vacuum liquid chromatography on RP-18 material with a gradient from $\mathrm{MeOH}: \mathrm{H}_{2} \mathrm{O}(10: 90)$ to $\mathrm{MeOH}$ in 10 steps and then DCM to yield 11 fractions. Fractions 7 and 8 were further separated by column chromatography on Sephadex LH-20 material to yield 27 fractions in each case. Fractions 7.11-13 and 8.12-15 were further separated by isocratic HPLC [column (1), MeOH: $\mathrm{H}_{2} \mathrm{O}(58: 42)$ ] to yield 10 and 8 fractions, respectively. Fractions 2 of both HPLC separations were combined because of their similar ${ }^{1} \mathrm{H}$ NMR spectra to yield $5.4 \mathrm{mg}$ of pure compound (1).

The pure metabolite (2) was isolated from a second ethyl acetate extract (7.2 g) obtained from $12 \mathrm{~L}$ of medium. The extract was fractionated by gradient RP-18 VLC [MeOH: $\mathrm{H}_{2} \mathrm{O}(10: 90)$ to $\mathrm{MeOH}$ in 10 steps and then DCM] to yield 11 fractions. Fraction 7 was further separated by column chromatography on Sephadex LH-20 material to yield 32 fractions. Fractions 13 and 14 were combined because of their similar ${ }^{1} \mathrm{H}$ NMR spectra, separated by isocratic HPLC [column (2), $\mathrm{MeOH}: \mathrm{H}_{2} \mathrm{O}$ (48:52)] and yielded pure compound (2) (3.6 mg).

Ascospiroketal A (1): white amorphous solid (5.4 mg); $[\alpha]^{23}{ }_{\mathrm{D}}+20$ (c 0.45 in $\left.\mathrm{MeOH}\right)$; UV $\lambda_{\max }(\mathrm{MeOH})(\log \varepsilon) 230 \mathrm{~nm}(4.09), 272 \mathrm{~nm} \mathrm{sh}$ (3.45); IR $v_{\max } 3416,2934,1708,1456,1362$, $1221,1061 \mathrm{~cm}^{-1}$; ${ }^{1} \mathrm{H}$ NMR [500 MHz, $\left(\mathrm{CD}_{3}\right)_{2} \mathrm{CO}$ ] and ${ }^{13} \mathrm{C} \mathrm{NMR}$ [300 $\mathrm{MHz},\left(\mathrm{CD}_{3}\right)_{2} \mathrm{CO}$ ], see Table S1; EI-MS m/z 438 (4), 320 (65), 272 (100), 197 (30), 179 (54); HR-EI-MS $m / z[\mathrm{M}+] 438.2259$ (calcd for $\mathrm{C}_{23} \mathrm{H}_{34} \mathrm{O}_{8} 438.2254$ ).

Ascospiroketal B (2): white amorphous solid (3.6 mg); $[\alpha]^{23}{ }_{\mathrm{D}}+3$ (c 0.33 in $\left.\mathrm{MeOH}\right)$; UV $\lambda_{\max }(\mathrm{MeOH})(\log \varepsilon) 225 \mathrm{~nm}(4.35), 275 \mathrm{~nm} \operatorname{sh}(3.73)$; IR $v_{\max }$ 3418, 2930, 1708, 1456, 1364, 1191, $1048 \mathrm{~cm}^{-1}$; ${ }^{1} \mathrm{H}$ NMR [500 MHz, $\left.\left(\mathrm{CD}_{3}\right)_{2} \mathrm{CO}\right]$ and ${ }^{13} \mathrm{C} \mathrm{NMR}$ [300 $\mathrm{MHz},\left(\mathrm{CD}_{3}\right)_{2} \mathrm{CO}$ ], see Table S2; EI-MS m/z 438 (0.5), 320 (32), 272 (74), 197 (100), 197 (100); HR-EIMS $m / z$ [M+] 438.2244 (calcd for $\mathrm{C}_{23} \mathrm{H}_{34} \mathrm{O}_{8} 438.2254$ ). 
Table S1. ${ }^{1} \mathrm{H}$ and ${ }^{13} \mathrm{C}$ NMR spectral data for Ascospiroketal A $(\mathbf{1})$ in $\left(\mathrm{CD}_{3}\right)_{2} \mathrm{CO}$.

\begin{tabular}{|c|c|c|c|c|c|c|}
\hline Position & $\mathrm{DEPT}^{a}$ & $\delta_{\mathrm{H}}$ mult. $(J \text { in } \mathrm{Hz})^{b}$ & $\delta_{\mathrm{C}}{ }^{a}$ & $\operatorname{COSY}^{b}$ & $\mathrm{HMBC}^{b, c}$ & $\mathrm{NOESY}^{b, d}$ \\
\hline 1 & $\mathrm{qC}$ & & 173.6 & & & \\
\hline 2 & $\mathrm{qC}$ & & 55.2 & & & \\
\hline 3 & $\mathrm{CH}$ & $4.38 \mathrm{~d},(3.5)$ & 91.2 & 4 & $4,6,16,17$ & $4,5,7^{\mathrm{e}}, 17$ \\
\hline 4 & $\mathrm{CH}$ & $4.73 \mathrm{q},(3.5)$ & 83.0 & 3,5 & $3,5,6,16$ & $3,5,16 b^{\mathrm{e}}, 17$ \\
\hline 5 & $\mathrm{CH}_{2}$ & $2.16 \mathrm{~d},(3.5)$ & 43.2 & 4 & $3,4,6$ & $3^{\mathrm{e}}, 4,7,16 \mathrm{a}^{\mathrm{e}}$ \\
\hline 6 & $\mathrm{qC}$ & & 117.0 & & & \\
\hline 7 & $\mathrm{CH}_{2}$ & $1.94 \mathrm{~m}$ & 38.1 & $8 \mathrm{a}, 8 \mathrm{~b}$ & 6,9 & \\
\hline \multirow[t]{2}{*}{8} & $\mathrm{CH}_{2}$ & a: $2.05 \mathrm{~m}$ & 32.2 & $7,8 b, 9$ & & 9 \\
\hline & & b: $1.70 \mathrm{~m}$ & & 7,9 & 9,10 & 10 \\
\hline 9 & $\mathrm{CH}$ & $4.46 \mathrm{q},(8.2)$ & 81.9 & $8 \mathrm{a}, 8 \mathrm{~b}, 10,11$ & $6,8,11$ & $5^{\mathrm{e}}, 7^{\mathrm{e}}, 8 \mathrm{a}, 8 \mathrm{~b}^{\mathrm{e}}, 10,11$ \\
\hline 10 & $\mathrm{CH}$ & $5.76 \mathrm{dd},(8.2,15.1)$ & 138.2 & 9,11 & $8,9,12$ & $8 b, 9,12,16 a$ \\
\hline 11 & $\mathrm{CH}$ & $6.45 \mathrm{dd},(11.0,15.1)$ & 126.0 & 10,12 & $9,12,13$ & $9,12,14,15^{\mathrm{e}}, 16 \mathrm{a}^{\mathrm{e}}, 18^{\mathrm{e}}$ \\
\hline 12 & $\mathrm{CH}$ & $6.06 \mathrm{t},(11.0)$ & 131.5 & $11,13,14$ & $10,11,14$ & $2^{\prime}, 10,13$ \\
\hline 13 & $\mathrm{CH}$ & $5.38 \mathrm{td},(11.0,7.9)$ & 126.9 & $11,12,14$ & $11,14,15$ & $12,14,15,18$ \\
\hline \multirow[t]{2}{*}{14} & $\mathrm{CH}_{2}$ & $\mathrm{a}: 2.47 \mathrm{~m}$ & 34.4 & $12,13,15$ & $12,13,15,18$ & $11,13,15,18$ \\
\hline & & b: $2.43 \mathrm{~m}$ & & $12,13,15$ & $12,13,15,18$ & $11,13,15,18$ \\
\hline 15 & $\mathrm{CH}$ & $4.90 \mathrm{sxt},(6.3)$ & 70.7 & 14,18 & $1^{\prime}, 13,14,18$ & $2^{\prime}, 11,13,14,18$ \\
\hline \multirow[t]{2}{*}{16} & $\mathrm{CH}_{2}$ & a: $4.63 \mathrm{~d},(7.9)$ & 73.5 & 17 & $1,2,17$ & 10,11 \\
\hline & & b: $3.47 \mathrm{~d},(7.9)$ & & 3 & $2,3,4,17$ & $4^{\mathrm{e}}, 17$ \\
\hline 17 & $\mathrm{CH}_{3}$ & $1.25 \mathrm{~s}$ & 21.0 & $16 b$ & $1,2,3,16$ & $3,4,16 b$ \\
\hline 18 & $\mathrm{CH}_{3}$ & $1.20 \mathrm{~d},(6.3)$ & 19.7 & 15 & 14,15 & $14 \mathrm{a}, 15$ \\
\hline $1^{\prime}$ & $\mathrm{qC}$ & & 175.0 & & & \\
\hline $2^{\prime}$ & $\mathrm{CH}$ & $2.34 \mathrm{p},(6.6)$ & 48.1 & $3^{\prime}, 4^{\prime}$ & $1^{\prime}, 3^{\prime}, 4^{\prime}, 5^{\prime}$ & $3^{\prime}, 4^{\prime}, 5^{\prime}, 12,15$ \\
\hline $3^{\prime}$ & $\mathrm{CH}$ & $3.89 \mathrm{p},(6.6)$ & 68.8 & $2^{\prime}, 5^{\prime}$ & $1^{\prime}, 2^{\prime}, 4^{\prime}, 5^{\prime}$ & $2^{\prime}, 4^{\prime}, 5^{\prime}$ \\
\hline $4^{\prime}$ & $\mathrm{CH}_{3}$ & $1.14 \mathrm{~d},(6.6)$ & 12.8 & $2^{\prime}, 3^{\prime}$ & $1^{\prime}, 2^{\prime}, 3^{\prime}$ & $2^{\prime}, 3^{\prime}$ \\
\hline $5^{\prime}$ & $\mathrm{CH}_{3}$ & $1.13 \mathrm{~d},(6.6)$ & 21.6 & $2^{\prime}, 3^{\prime}$ & 1', 2', 3' & 2', 3' \\
\hline
\end{tabular}

Figure S1. $\quad 500 \mathrm{MHz}{ }^{1} \mathrm{H}$ NMR spectrum of Ascospiroketal A (1) in $\left(\mathrm{CD}_{3}\right)_{2} \mathrm{CO}$.

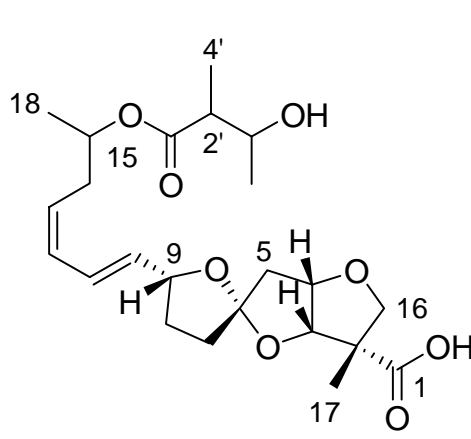
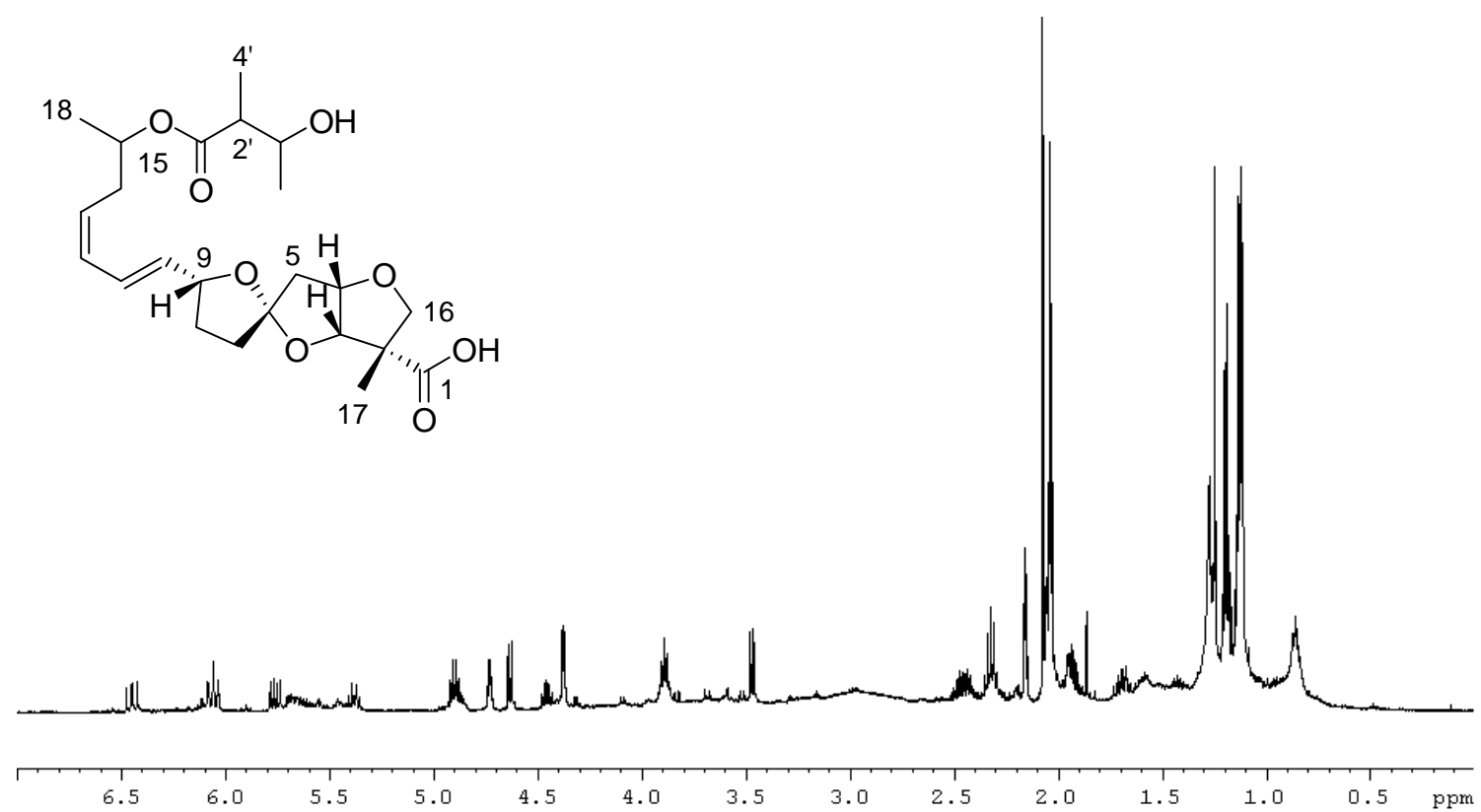
Figure S2. $\quad 75 \mathrm{MHz}{ }^{13} \mathrm{C}$ NMR and $75 \mathrm{MHz}$ DEPT 135 spectra of Ascospiroketal A (1) in $\left(\mathrm{CD}_{3}\right)_{2} \mathrm{CO}$.
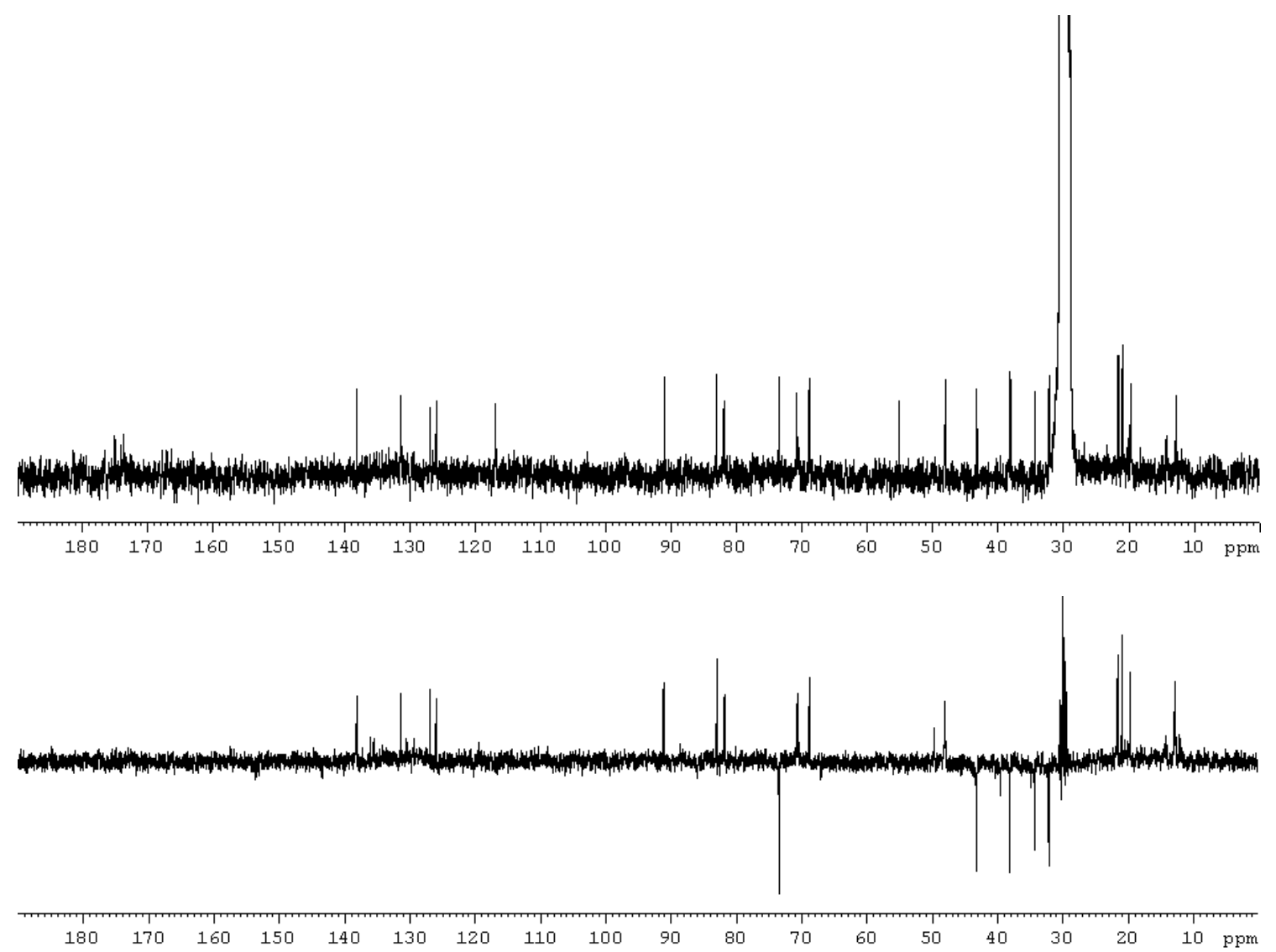
Figure S3. $\quad 500 \mathrm{MHz}{ }^{1} \mathrm{H}-{ }^{1} \mathrm{H}-\mathrm{COSY}$ spectrum of Ascospiroketal A (1) in $\left(\mathrm{CD}_{3}\right)_{2} \mathrm{CO}$.

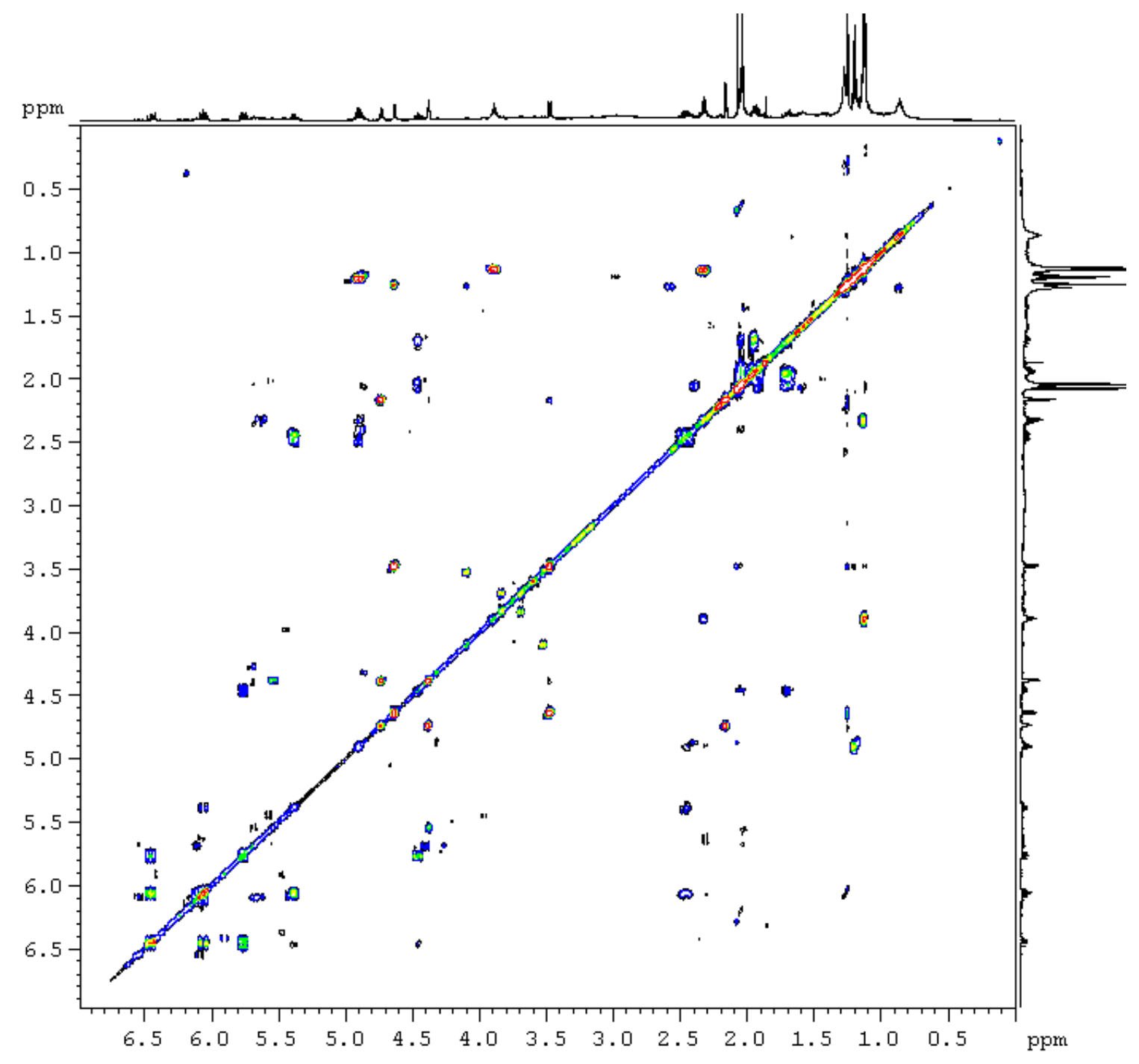


Figure S4. $500 \mathrm{MHz}$ HSQC spectrum of Ascospiroketal A (1) in $\left(\mathrm{CD}_{3}\right)_{2} \mathrm{CO}$.

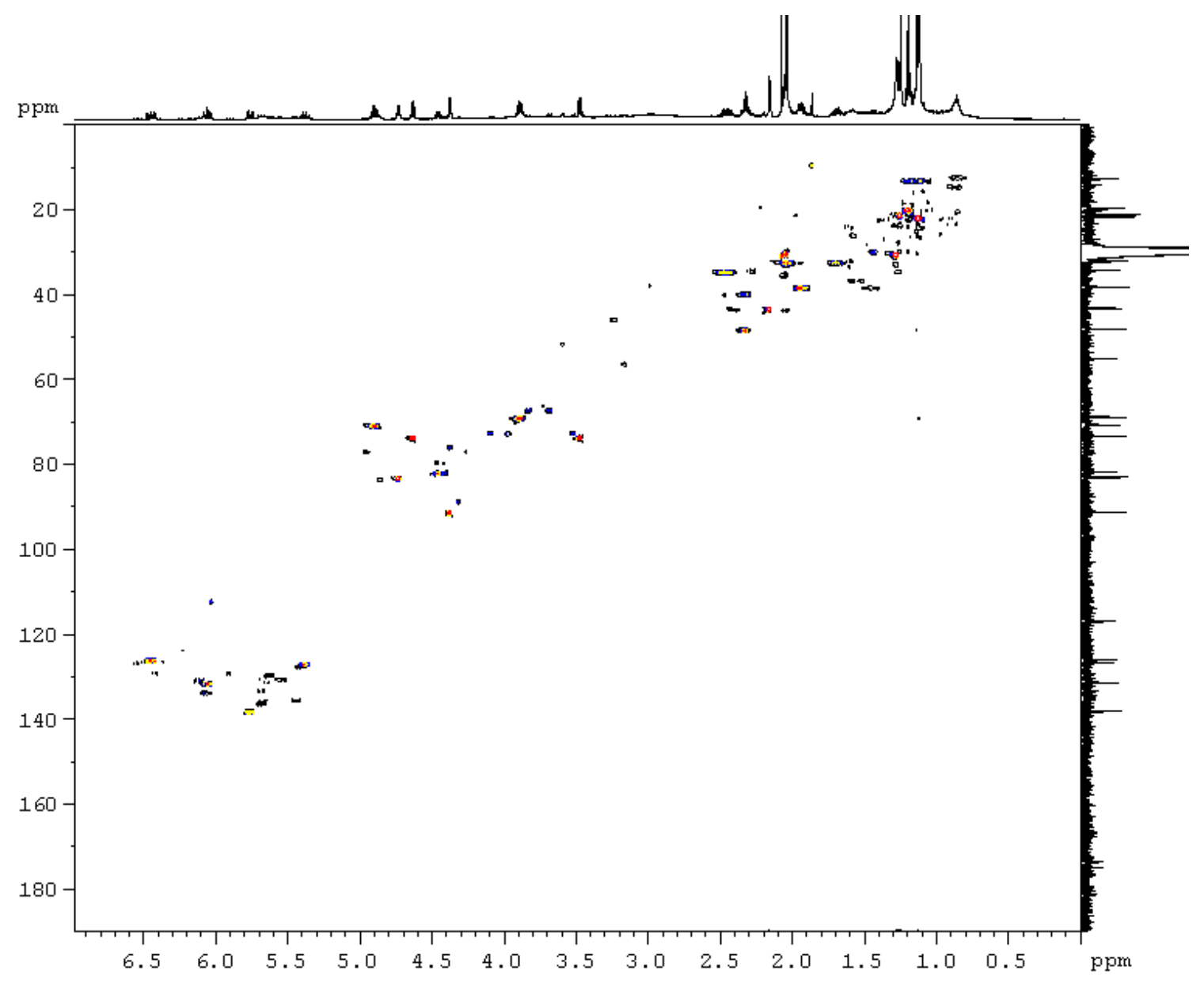


Figure S5. $\quad 500 \mathrm{MHz} \mathrm{HMBC}$ spectrum of Ascospiroketal A (1) in $\left(\mathrm{CD}_{3}\right)_{2} \mathrm{CO}$.

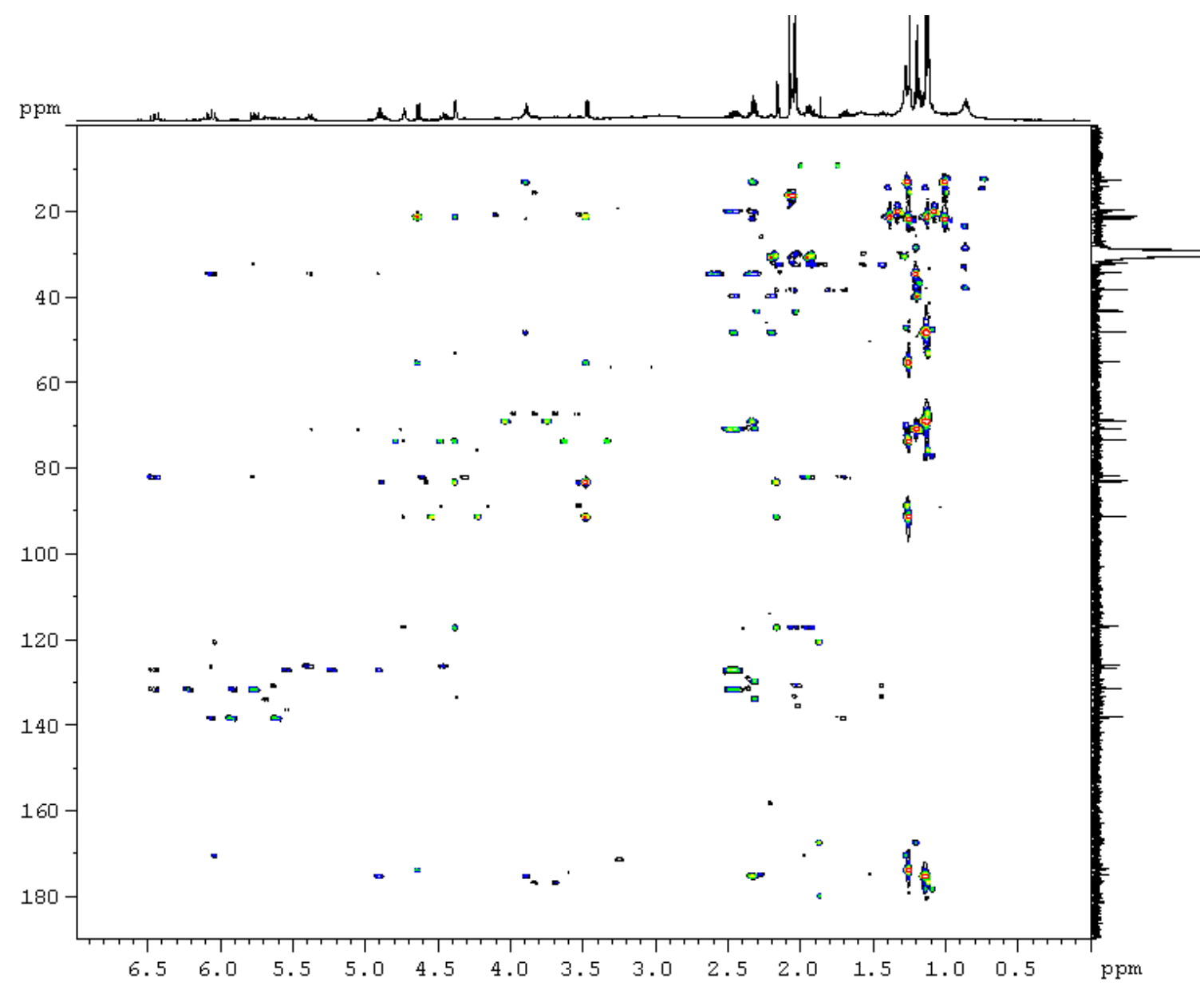


Figure S6. $\quad 500 \mathrm{MHz}$ NOESY spectrum of Ascospiroketal A (1) in $\left(\mathrm{CD}_{3}\right)_{2} \mathrm{CO}$.

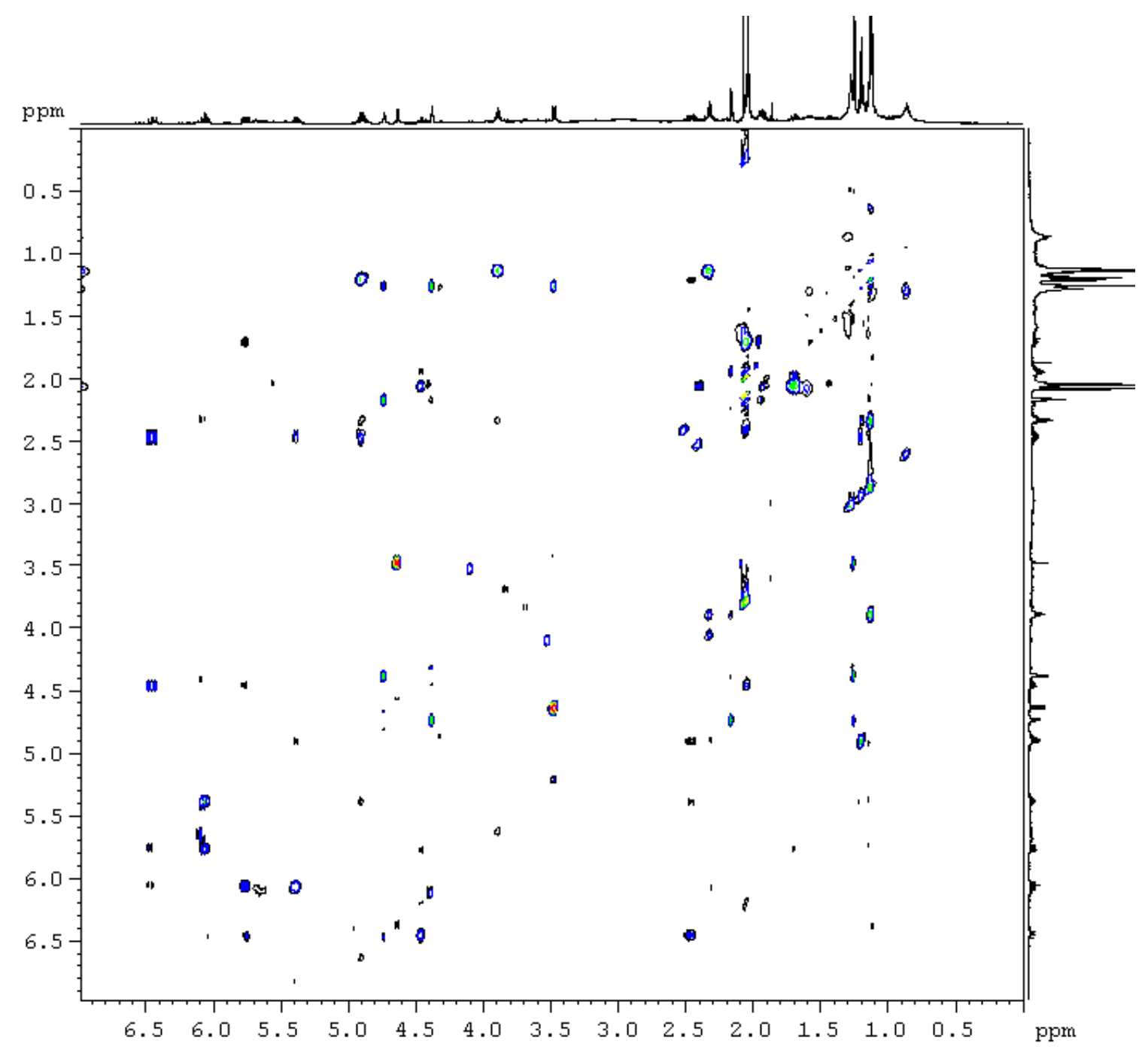


Figure S7. 3D drawing of Ascospiroketal A (1) with selected NOESY correlations.

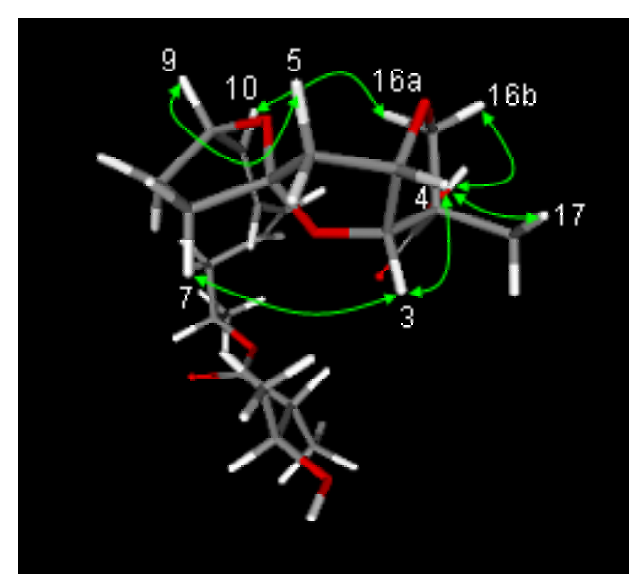

3D drawing of Ascospiroketal A (1). Selected NOEs from selective gradient NOE experiments (green arrows). Configuration at C-2', C-3' and C-15 proposed. The 3D model was drawn on a silicon graphics $\mathrm{O} 2$ workstation using the Cerius ${ }^{2} 4.0$ (MSI) software package. 
Table S2. ${ }^{1} \mathrm{H}$ and ${ }^{13} \mathrm{C}$ NMR spectral data for Ascospiroketal B (2) in $\left(\mathrm{CD}_{3}\right)_{2} \mathrm{CO}$.

\begin{tabular}{|c|c|c|c|c|c|c|}
\hline Position & $\mathrm{DEPT}^{a}$ & $\delta_{\mathrm{H}}$ mult. $(J \text { in } \mathrm{Hz})^{b}$ & $\delta_{\mathrm{C}}{ }^{a}$ & $\operatorname{COSY}^{b}$ & $\mathrm{HMBC}^{b, c}$ & $\mathrm{NOESY}^{b, d}$ \\
\hline 1 & $\mathrm{qC}$ & & 180.1 & & & \\
\hline 2 & $\mathrm{qC}$ & & 51.6 & & & \\
\hline 3 & $\mathrm{CH}$ & $4.60 \mathrm{~d},(4.4)$ & 84.1 & 4 & $1,4,16$ & $4,5 \mathrm{a}, 16,17$ \\
\hline 4 & $\mathrm{CH}$ & $5.10 \mathrm{ddd},(2.2,4.4,6.9)$ & 82.2 & $3,5 \mathrm{a}, 5 \mathrm{~b}$ & $1,3,5,6$ & $3,3^{\prime}, 5 \mathrm{a}, 5 \mathrm{~b}^{e}, 15^{e}, 16 \mathrm{a}$ \\
\hline \multirow[t]{2}{*}{5} & $\mathrm{CH}_{2}$ & a: $2.58 \mathrm{dd},(6.9,14.8)$ & 42.9 & 4 & 3,6 & $3^{e}, 4$ \\
\hline & & $\mathrm{b}: 2.27 \mathrm{dd},(2.2,14.8)$ & & 4 & $3,4,6$ & 7 \\
\hline 6 & $\mathrm{qC}$ & & 116.0 & & & \\
\hline 7 & $\mathrm{CH}_{2}$ & $2.10 \mathrm{~m}$ & 35.6 & $8 a, 8 b$ & $6,8,9$ & \\
\hline \multirow[t]{2}{*}{8} & $\mathrm{CH}_{2}$ & $\mathrm{a}: 2.21 \mathrm{~m}$ & 31.8 & 7,9 & $6,9,10$ & 7,9 \\
\hline & & $\mathrm{b}: 1.67 \mathrm{~m}$ & & 7,9 & $6,7,9,10$ & $7,9,10$ \\
\hline 9 & $\mathrm{CH}$ & $4.55 \mathrm{q},(6.9)$ & 79.6 & $8,10,11$ & $8,10,11$ & $8 \mathrm{a}, 8 \mathrm{~b}^{e}, 10,11$ \\
\hline 10 & $\mathrm{CH}$ & $5.71 \mathrm{dd},(6.9,15.1)$ & 135.4 & 9,11 & $8,9,12$ & $8 b, 9,12$ \\
\hline 11 & $\mathrm{CH}$ & $6.58 \mathrm{dd},(11.0,15.1)$ & 126.7 & 10,12 & $9,12,13$ & $9,12,14,15^{e}$ \\
\hline 12 & $\mathrm{CH}$ & $6.11 \mathrm{t},(11.0)$ & 131.2 & 11,13 & $10,11,14$ & $2^{e}, 10,11^{e}, 13$ \\
\hline 13 & $\mathrm{CH}$ & $5.45 \mathrm{~m}$ & 127.6 & $11,12,14$ & $11,14,15$ & $12,14,15,18$ \\
\hline \multirow[t]{2}{*}{14} & $\mathrm{CH}_{2}$ & $\mathrm{a}: 2.52 \mathrm{~m}$ & 34.5 & $12,13,15$ & $12,13,15,18$ & $11,13^{e}, 18$ \\
\hline & & $\mathrm{b}: 2.42 \mathrm{~m}$ & & $12,13,15$ & $12,13,15,18$ & $11,13,15,18$ \\
\hline 15 & $\mathrm{CH}$ & $4.91 \mathrm{sxt},(6.3)$ & 70.6 & 14,18 & $1,, 13,14,18$ & $2^{\prime}, 13,14 \mathrm{a}, 14 \mathrm{~b}, 18$ \\
\hline \multirow[t]{2}{*}{16} & $\mathrm{CH}_{2}$ & a: $3.67 \mathrm{~d},(10.4)$ & 68.0 & & $1,2,3,17$ & $3,4,17$ \\
\hline & & b: $3.64 \mathrm{~d},(10.4)$ & & & $1,2,3,17$ & $3,4,17$ \\
\hline 17 & $\mathrm{CH}_{3}$ & $1.09 \mathrm{~s}$ & 14.0 & & $1,2,3,16$ & $3,16 a^{e}, 16 b$ \\
\hline 18 & $\mathrm{CH}_{3}$ & $1.22 \mathrm{~d},(6.3)$ & 19.8 & 15 & 14,15 & 15 \\
\hline 1 ' & $\mathrm{qC}$ & & 175.0 & & & \\
\hline $2^{\prime}$ & $\mathrm{CH}$ & $2.33 \mathrm{p},(6.6)$ & 48.1 & $3^{\prime}, 4^{\prime}$ & $1^{\prime}, 3^{\prime}, 4^{\prime}, 5^{\prime}$ & $3^{\prime}, 4^{\prime}, 5^{\prime}, 10,12,15$ \\
\hline 3 , & $\mathrm{CH}$ & $3.89 \mathrm{p},(6.6)$ & 68.9 & $2^{\prime}, 5^{\prime}$ & $1^{\prime}, 2^{\prime}, 4^{\prime}, 5^{\prime}$ & $2^{\prime}, 4^{\prime}, 5^{\prime}$ \\
\hline $4^{\prime}$ & $\mathrm{CH}_{3}$ & $1.14 \mathrm{~d},(6.6)$ & 12.9 & 2 & $2^{\prime}, 3^{\prime}, 5^{\prime}$ & $2^{\prime}, 3^{\prime}$ \\
\hline 5 & $\mathrm{CH}_{3}$ & $1.13 \mathrm{~d},(6.6)$ & 21.7 & 3 , & $1,2,3$, & $2,, 3$, \\
\hline
\end{tabular}

${ }^{a}$ Recorded at $75 \mathrm{MHz} .{ }^{b}$ Recorded at $500 \mathrm{MHz} .{ }^{c}$ Data reported for experiment optimized for either $5 \mathrm{~Hz}$ or $8 \mathrm{~Hz}$. ${ }^{d} \mathrm{Data}$ obtained from 2D NOESY experiments and selective NOESY experiments. ${ }^{e}$ Weak correlation.

Figure S8. $\quad 500 \mathrm{MHz}{ }^{1} \mathrm{H}$ NMR spectrum of Ascospiroketal B (2) in $\left(\mathrm{CD}_{3}\right)_{2} \mathrm{CO}$.

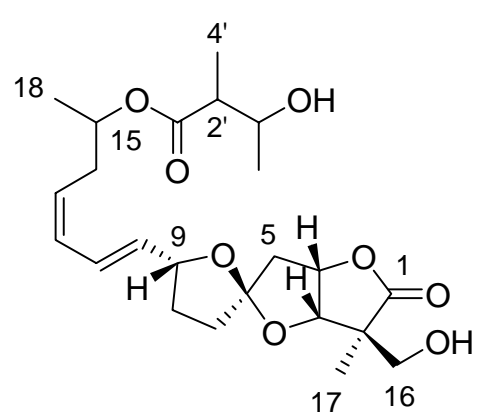
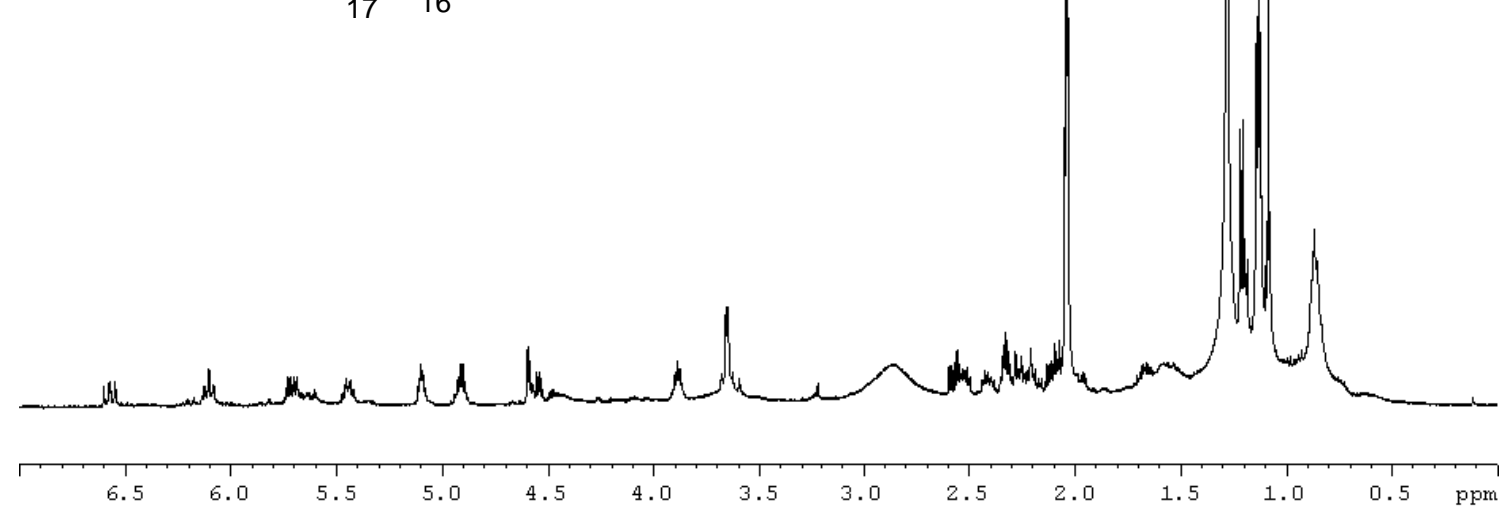
Figure S9. $\quad 75 \mathrm{MHz}{ }^{13} \mathrm{C}$ NMR and $75 \mathrm{MHz}$ DEPT 135 spectrum of Ascospiroketal B (2) in $\left(\mathrm{CD}_{3}\right)_{2} \mathrm{CO}$.
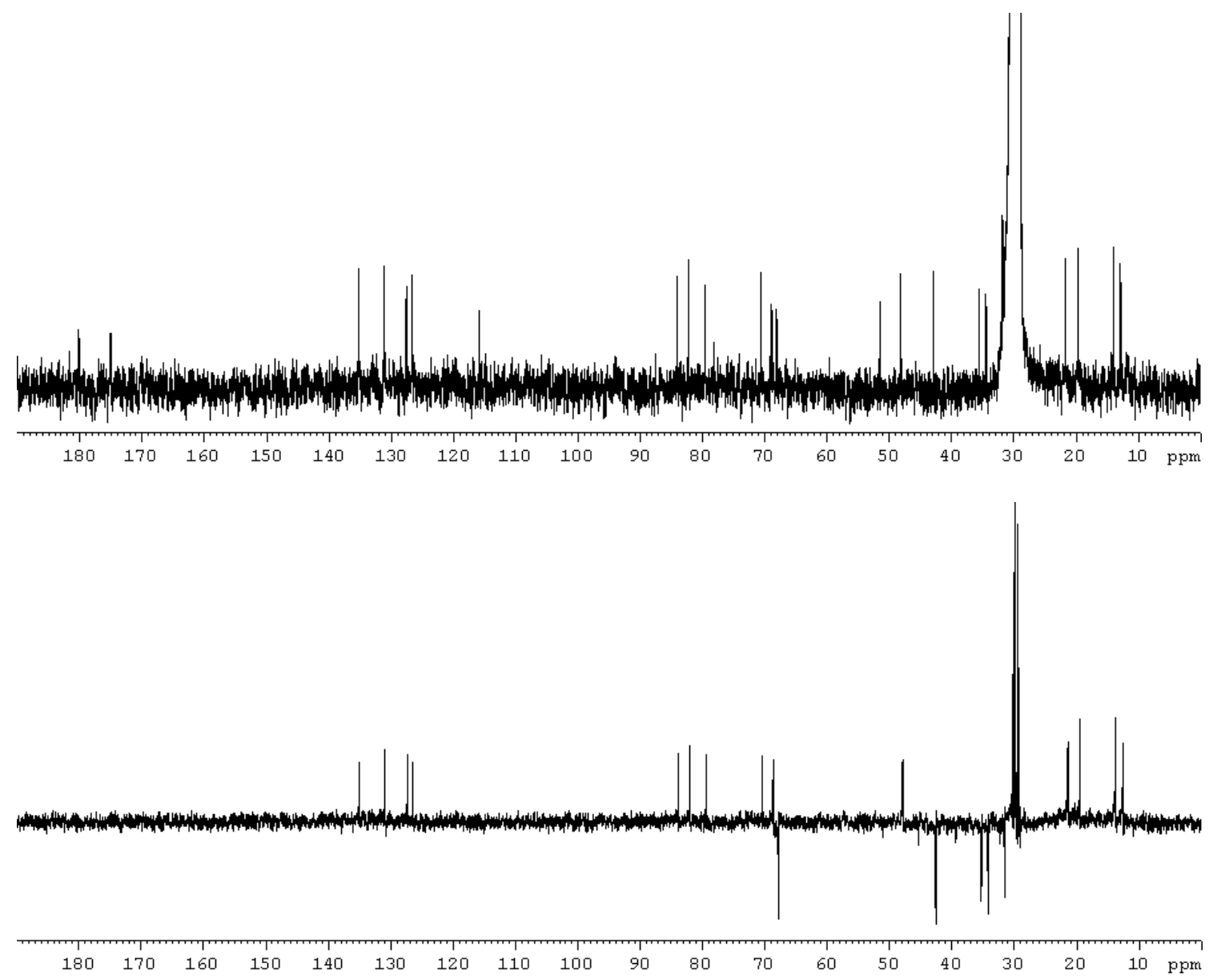
Figure S10. $500 \mathrm{MHz}{ }^{1} \mathrm{H}-{ }^{1} \mathrm{H}-\mathrm{COSY}$ spectrum of Ascospiroketal B (2) in $\left(\mathrm{CD}_{3}\right)_{2} \mathrm{CO}$.

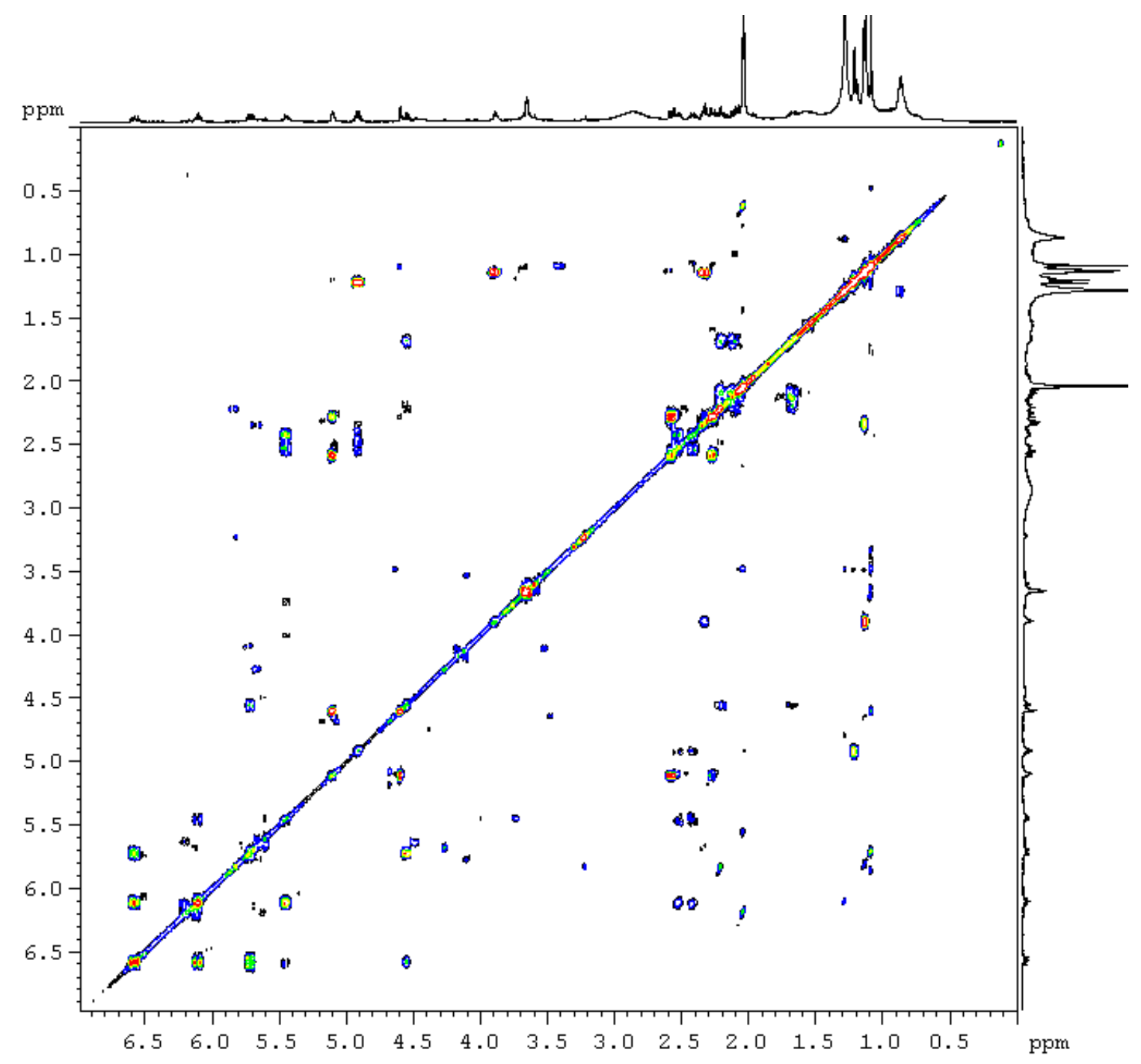


Figure S11. $500 \mathrm{MHz}$ HSQC spectrum of Ascospiroketal B (2) in $\left(\mathrm{CD}_{3}\right)_{2} \mathrm{CO}$.

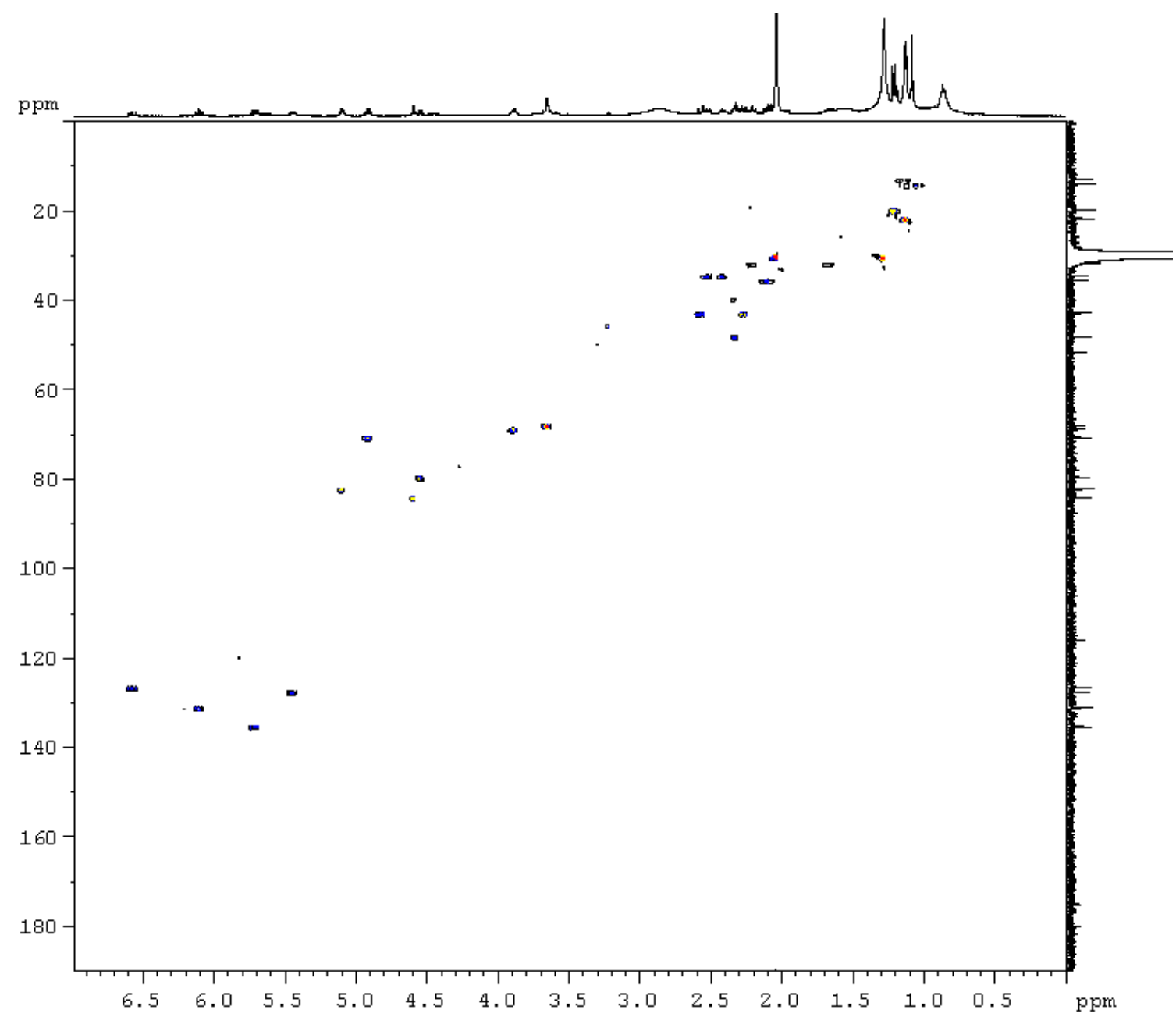


Figure S12. $500 \mathrm{MHz} H \mathrm{HBC}$ spectrum of Ascospiroketal B (2) in $\left(\mathrm{CD}_{3}\right)_{2} \mathrm{CO}$.

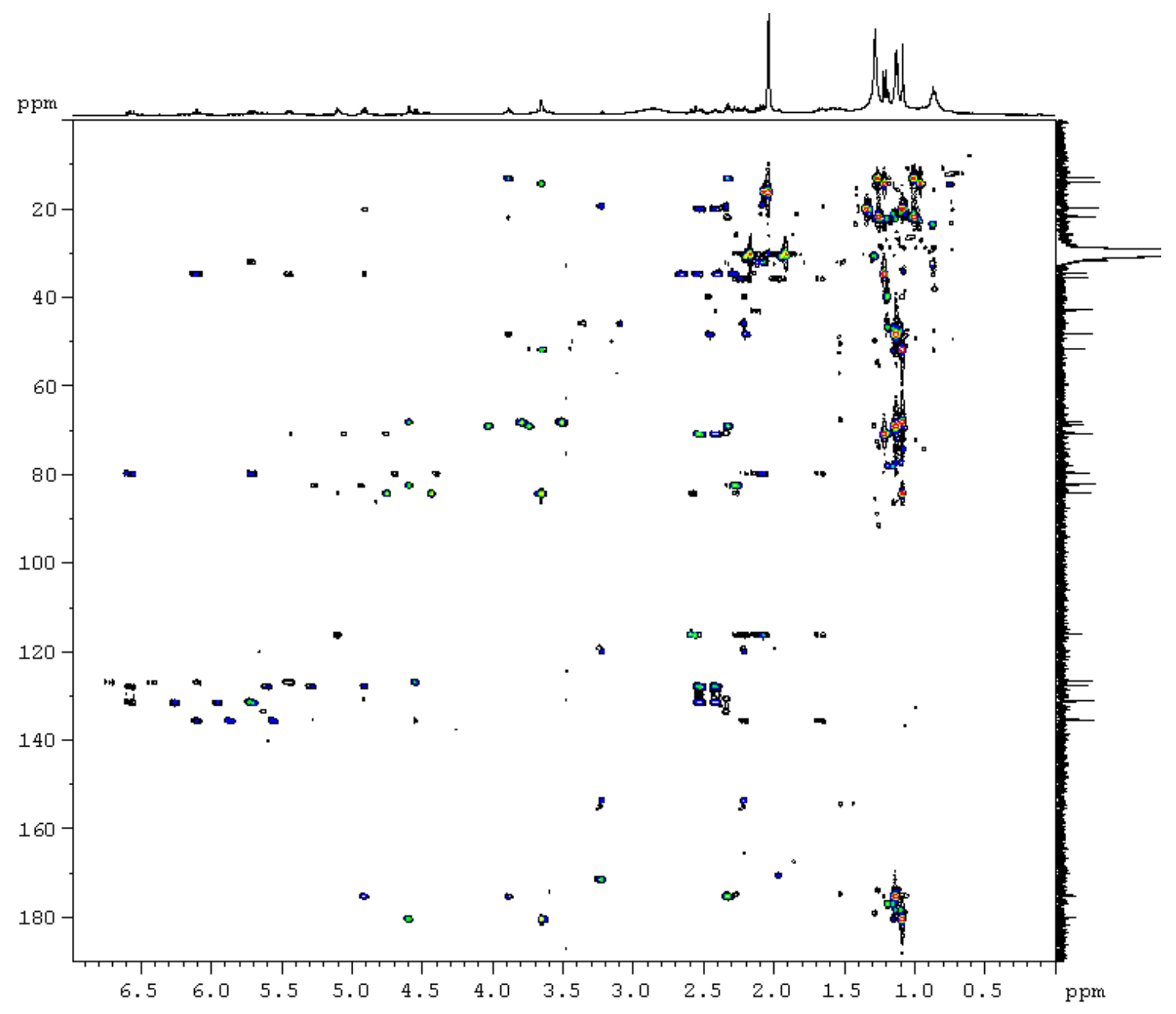


Figure S13. $500 \mathrm{MHz}$ NOESY spectrum of Ascospiroketal B (2) in $\left(\mathrm{CD}_{3}\right)_{2} \mathrm{CO}$.

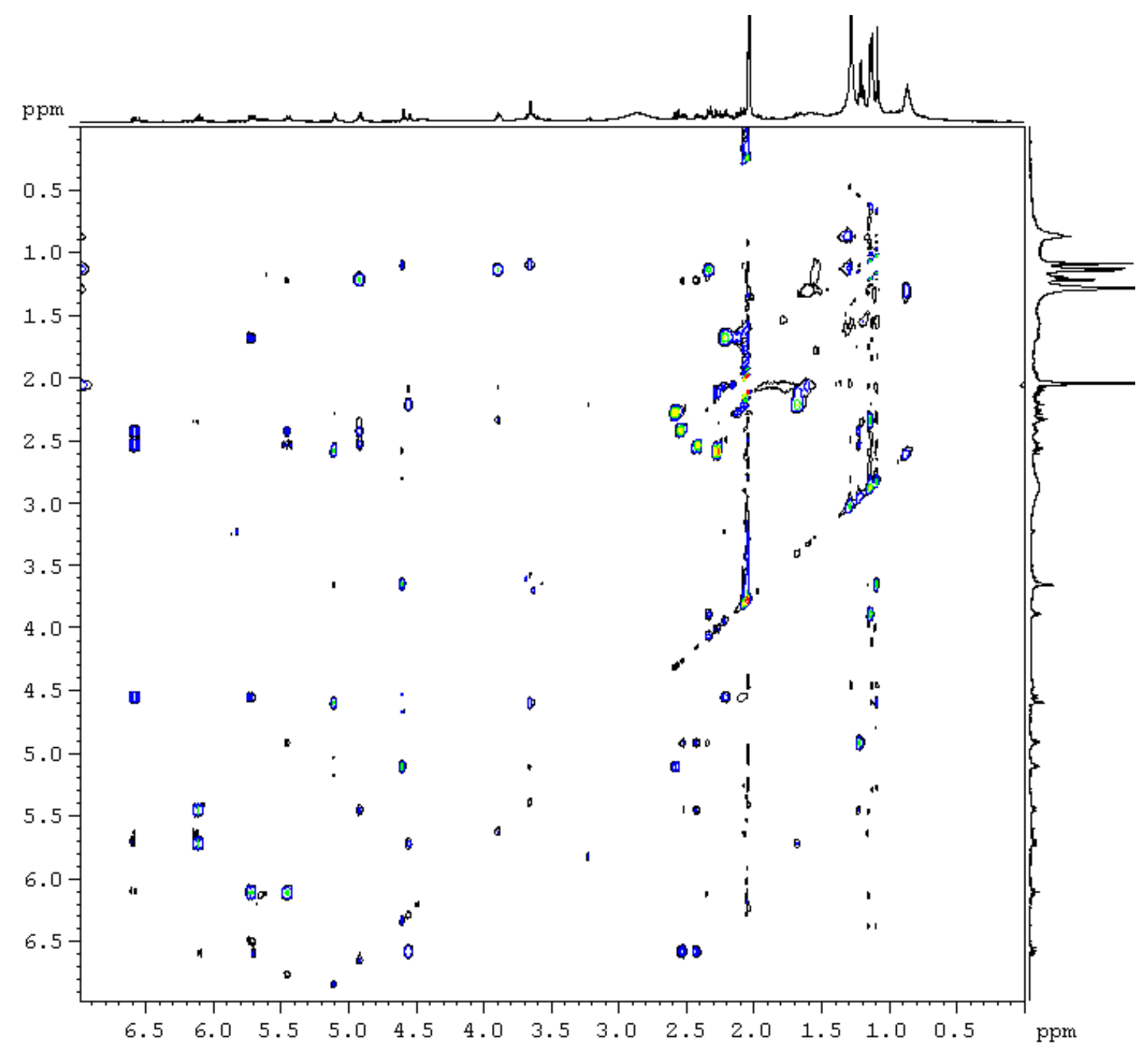


Figure S14. 3D drawing of Ascospiroketal B (2) with selected NOESY correlations.

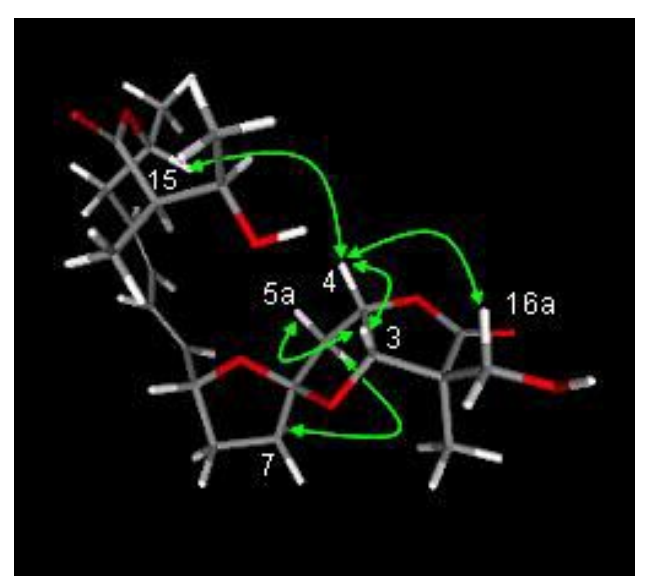

3D drawing of Ascospiroketal B (2). Selected NOEs from selective gradient NOE experiments (green arrows). Configuration at C-2', C-3' and C-15 proposed. The 3D model was drawn on a silicon graphics $\mathrm{O} 2$ workstation using the Cerius ${ }^{2} 4.0$ (MSI) software package. 